\title{
An Improved SSD for small target detection
}

\begin{abstract}
Xiang Li
Key Laboratory of Opt-Electronic Information Processing, Chinese Academy of Sciences, Shenyang 110016, China; Shenyang Institute of Automation, Chinese Academy of Sciences, Shenyang 110016, China; Institutes for Robotics and Intelligent Manufacturing, Chinese Academy of Sciences, Shenyang 110169; University of Chinese Academy of Sciences, Beijing 100049, China; The Key Laboratory of Image Understanding and Computer Vision, Shenyang, Liaoning 110016
\end{abstract}

\begin{abstract}
SSD is one of heuristic one-stage target detection approaches. Although it has got impressive results in general target detection, it still struggles in small-size object detection and precise location. In this paper, we proposed an improved SSD which forces on the small-size target detection. We include a shallow and high resolution feature into the hierarchical detection feature which are used for prediction. Then, we fuse the detection features (including the shallow and high resolution one) as a feature pyramid through some $1 \times 1$ convolution layers and unsample operations to pass information from deep features to the shallow ones, aiming to enrich the semantic information of the shallow features. To make the network easier to converge, we add a L2 normalization to the bottom detection feature of the feature pyramid to make a norm balance between each pyramid feature. The experimental results on the VEDAI dataset show that the proposed method has obtained impressive progress than the original SSD for the small targets detection.
\end{abstract}

\section{CCS CONCEPTS}

- Computing methodologies; $\bullet$ Artificial intelligence; $\bullet$ Computer vision; $\bullet$ Computer vision problems; $\bullet$ Object detection;

\section{KEYWORDS}

Convolution neural network, Target detection, SSD, Small targets

\section{ACM Reference Format:}

Xiang Li and Haibo LuoX. 2021. An Improved SSD for small target detection. In 2021 6th International Conference on Multimedia and Image Processing (ICMIP 2021), fanuary 08-10, 2021, Zhuhai, China. ACM, New York, NY, USA, 5 pages. https://doi.org/10.1145/3449388.3449391

\footnotetext{
*X IANG LI was born in Ningxia, in 1993 . He is currently pursuing the Ph.D. degree at the University of Chinese Academy of Science. His current research includes deep learning, target detection, and infrared target detection.

Permission to make digital or hard copies of all or part of this work for personal or classroom use is granted without fee provided that copies are not made or distributed for profit or commercial advantage and that copies bear this notice and the full citation on the first page. Copyrights for components of this work owned by others than ACM must be honored. Abstracting with credit is permitted. To copy otherwise, or republish, to post on servers or to redistribute to lists, requires prior specific permission and/or a fee. Request permissions from permissions@acm.org.

ICMIP 2021, January 08-10, 2021, Zhuhai, China

(C) 2021 Association for Computing Machinery.

ACM ISBN 978-1-4503-8916-7/21/01 . \$ \$15.00

https://doi.org/10.1145/3449388.3449391
}

\author{
Haibo LuoX* \\ Key Laboratory of Opt-Electronic Information Processing, \\ Chinese Academy of Sciences, Shenyang 110016, China; \\ Shenyang Institute of Automation, Chinese Academy of \\ Sciences, Shenyang 110016, China; Institutes for Robotics \\ and Intelligent Manufacturing, Chinese Academy of \\ Sciences, Shenyang 110169; University of Chinese \\ Academy of Sciences, Beijing 100049, China; The Key \\ Laboratory of Image Understanding and Computer Vision, \\ Shenyang, Liaoning 110016
}

\section{INTRODUCTION}

Small target detection in remote sensing images is one of the research hotpots in computer vison and is widely used in military reconnaissance, intelligence transportation and intelligence surveillance [1], [2]. Because of the small size of the targets and the complex background, small targets detection is typically a very challenging task.

With the development of deep learning, some effective algorithms have been proposed for target detection such as R-CNN [3], Fast-RCNN [4], Faster-RCNN [5], SSD [6], YOLO [7], YOLO 9000 [8], OverFeat [9]. Although these algorithms have made significant progress compare to the traditional ones, they are still not ideal in small targets detection because they are all designed for general objects. Compared to the general targets, the small targets are always inconspicuous and have nor so many details like texture and shape. In addition, the small targets are easily 'polluted' by the surrounding pixels like shadow. So it is more difficult to classify with such less semantic information. To solve this problem, some works have been proposed such as FPN [10], DSSD [11], PANet [12]. They used feature pyramids to enrich semantic information of the shallow but high resolution features to boost the classify performance and ensure location precision simultaneously.

In this paper, we propose an improved SSD for small targets detection. Firstly, we include the $3^{\text {rd }}$ feature of the $3^{\text {rd }}$ convolution block (Conv3_3) of the VGG nets as one of the source features to make the improved SSD more suitable for small target detection. Because high resolution feature is beneficial to alleviate the influence from irrelevant adjacent background. Then, we adopt feature pyramid produced by fusing deep feature with the shallow feature to enrich the semantic information of the shallow feature map. In addition, we add a L2 normalization layer to the Conv3_3 layer to make the network easier to converge. Finally, we do comparative experiments on VEDAI [13] dataset to verify the performance of our improved SSD. The experiments results show that the proposed method outperforms than the original SSD for small target detection.

\section{METHODOLOGY}

In this section, we introduce our method in details. Frist, we adopt the feature pyramid which is made by fusing the sources features to replace the detection features that are used to classification and localization in SSD. This modification can enrich the semantic 


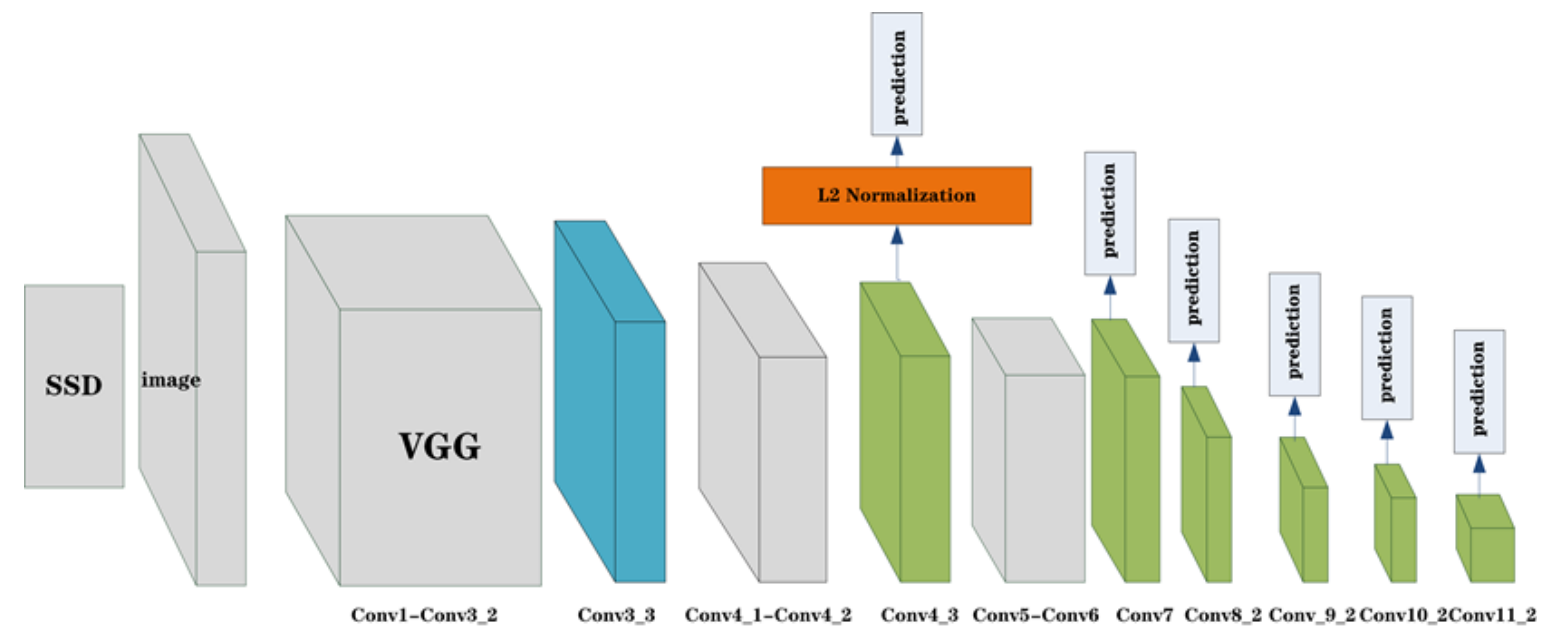

Figure 1: The green boxes are the source features of original SSD. In addition to this, we also include the shallow blue box Conv3_3 feature which has higher revolution as one of our source features.

information of the shallow feature and boost the classification performance. Then, to make the original SSD to fit for the small target detection, we enlarge the resolution of the lowest layer feature of the feature pyramid. Finally, to make the training process more easily to converge, we add a L2 normalization layer for the lowest feature of the feature pyramid to balance to the gradient proportion between each source features.

\subsection{Source features}

The SSD uses a conv4_3 of VGG nets [14] as the bottom layer of the featurized image pyramid. Thus it misses the opportunity to reuse the higher-resolution feature maps of the hierarchy which are important for detecting small objects. To make the SSD more suitable for small target tasks, we leverage the shallow, high resolution feature maps as the source features in our improved SSD. Small targets are more sensitive to the location error than the large targets and minor mistakes can make the value of jaccard overlap vary severely. High resolution features have advantage over localization than the low resolution ones and obtain more precise information about the location of the targets. Therefore, in the proposed method, we adopt the conv3_3 of VGG net as the bottom layer of the feature pyramid, and abandon the conv11_2 layer in our top-down feature pyramid which will be introduced in next section, as shown in Figure 1

\subsection{Top-down feature pyramid}

Benefit from the hierarchical construction with the feature maps at different resolution, the original SSD can detect different size targets in different source features. However, because of the inherent property of the deep neural network, the semantic information in shallow features with large resolution is not as rich as the deep ones. According to the [8], a feature pyramid that combines information from different feature maps has strong semantics at all scales. In this paper, we adopt a feature pyramid which is produced by fusing our source features in our improved SSD. To achieve this goal and make the features from different layers have the same size, we adopt a top-down pathway which is constants of upsampling and $1 \times 1$ convolutional layer, as shown in Figure2. First, we do the forward pass and pick seven features, as the blue and green boxes shown in Figure 1, as the source features. Then, we regard the top source feature Conv11_2 which has the lowest resolution as the anchor feature and upsample it, and then utilize the $1 \times 1$ convolutional layer so that the upsampled feature will have same width, height and channels as the next source feature. Furthermore, we fuse the obtained feature with the next source feature using element-wise summation (EWS). Finally, we make the fused feature obtained through EWS as a new anchor feature and perform the same operation like above with the next source feature which has higher resolution. With the above process, we can obtain a feature pyramid fused by different source features

\subsection{L2 normalization layer}

The original SSD adds a L2 normalization layer [15] at the beginning of the conv4_3 prediction branch, as shown in Figure 1. Through the process of the experiment, we find that the mean value of the conv4_3 feature is significant larger than other 5 source features'. If we delete this L2 normalization layer, it's hard to make net converge. So the main function of the L2 normalization layer on conv4_3 layer is to reduce the corresponding feature's norm and balance the gradient values from various feature in the optimization process. In our improved SSD, we include the conv3_3 feature as one of the source features and we find the same phenomenon that the mean value of this feature is two orders of magnitude larger than other source features. Therefore, we add a L2 normalization layer to the conv3_3 feature to scale the feature norm at each location in the feature map to 20 and learn the scale during back propagation. This L2 normalization layer has a coefficient matrix $W_{l 2}$ which has the same width and height as its corresponding feature. The normalization process is shown as equation (1):

$$
x_{s_{\text {norm }}}=\frac{x_{s} \times W_{l 2_{s}}}{\sqrt{\sum_{i=1}^{C} x_{s_{i}}^{2}}+\text { eps }}
$$




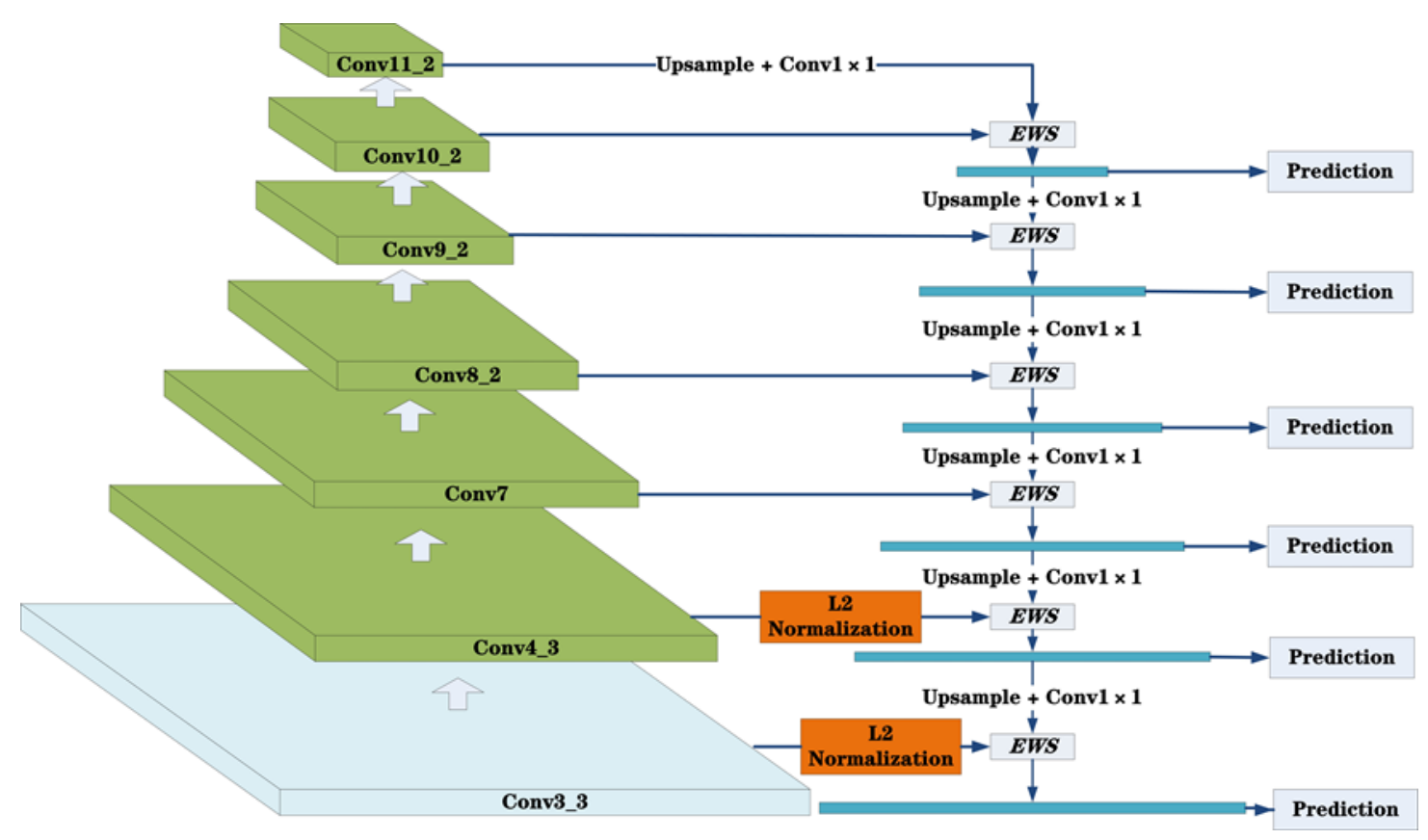

Figure 2: The proposed feature pyramid is consist of the blue box features in the middle column which are produced by fusing our source features on the left column. The orange boxes are the L2 normalization layer to scale the norm of the corresponding feature.

$$
s \in\{1,2 \ldots w \times h\}
$$

where $x_{s}$ is one of the channel vector of the source feature $x . W_{l 2_{s}}$ is the element of $W_{l 2}$ corresponding to $x_{s} . C$ is the channels number of the feature, and $w$ and $h$ are width, height of the current feature respectively. eps is a small value and can avoid the denominator equals zero and we set eps $=10^{-10}$. In this way we can make the norm of con3_3 feature has the same order of magnitudes as other source features.

\section{EXPERIMENT}

In this section, we will conduction comparative experiments to verify the performance of our improvement.

\subsection{VEDAI dataset}

To evaluate the performance of our improved SSD, we do experiments on VEDAI dataset to compare the original SSD with ours. VEDAI dataset is for vehicle detection in Aerial Imagery and the targets in it are all small targets. The original large aerial images are segmented into $1024 \times 1024$, containing various vehicles and backgrounds. To make the targets smaller, the images are down-sampled to $512 \times 512$. In VEDIA dataset, there are nine different categories of vehicles, namely the 'airplane', 'boat', 'camping', 'car', 'pickup', 'tractor', 'truck', 'vans', and 'others'.

\subsection{Implementation Details}

We adopt data augmentation which is the same as the original SSD. Each training image is randomly sampled by one of the following options:
1. Use the entire original input image.

2. Sample a patch whose minimum jaccard overlap with the objects is $0.1,0.3,0.5,0.7$, or 0.9 .

3. Randomly sample a patch.

The experiments are implemented with pytorch 1.0.0. The models are trained by stochastic gradient descent (SGD). We use the $2 \times 10^{-4}$ learning rate for first $20 \mathrm{k}$ iterations, then continue training for $15 \mathrm{k}$ iterations and the next $10 \mathrm{k}$ iterations with $2 \times 10^{-5}$ and $2 \times 10^{-6}$ learning rate, respectively. The weight decay, momentum and batch size were set by $5 \times 10^{-4}, 0.9,8$, respectively. The models are trained on one NVIDIA GeForce GTX TITAN X.

\subsection{Evaluation Metrics}

The mean average precision $(\mathrm{mAP})$ is used to evaluate the performance of the models. We evaluated the detectors with the common performance metric in target detection if Intersection over Union (IOU) with the ground truth bounding box is greater than 0.5 .

\subsection{Quantitative Results}

We use the mean average precision (mAP) to evaluate the performance of the models. The experimental results on VEDAI dataset between improved SSD and the original SSD are shown in Table 1. The comparative experiments are conducted with two input size 300 and 512, since the designed input size of original SSD was 300 and the image size in the VEDAI dataset is 512. As shown in Table 1, improved SSD achieves $66.8 \% \mathrm{mAP}$ on VEDAI dataset with the input size 512 and is the best one among the four trained models. The $\mathrm{mAP}$ has been improved by 15.9 percentage points with the input size 300 and 12.5 percentage points with the input size 512 
Table 1: The comparative results of the original SSD and the improved one

\begin{tabular}{lllllllllll}
\hline Detector & $\mathrm{mAP}(\%)$ & car & track & tractor & camping & vans & pickup & boat & airplane & others \\
\hline SSD-300 & 41.0 & 31.3 & 44.6 & 41.4 & 38.3 & 31.8 & 31.9 & 20.6 & 100.0 & 29.0 \\
Ours-300 & 56.9 & 52.6 & 61.6 & 62.1 & 59.6 & 48.5 & 52.7 & 32.1 & 100.0 & 42.6 \\
SSD-512 & 44.3 & 35.3 & 39.6 & 34.2 & 52.2 & 40.7 & 47.7 & 29.4 & 100.0 & 19.7 \\
Ours-512 & 66.8 & $\mathbf{6 1 . 4}$ & $\mathbf{6 3 . 9}$ & $\mathbf{7 0 . 4}$ & $\mathbf{6 8 . 8}$ & $\mathbf{7 8 . 8}$ & $\mathbf{6 2 . 7}$ & $\mathbf{4 7 . 7}$ & 100.0 & $\mathbf{4 7 . 5}$ \\
\hline
\end{tabular}

Table 2: The results of each algorithm on VEDAI dataset

\begin{tabular}{lllll}
\hline Detector & Faster RCNN & YOLO V2 & YOLO V3 & improved SSD \\
\hline Input & 512 & 512 & 512 & 512 \\
mAP $(\%)$ & 64.7 & 50.3 & 62.8 & $\mathbf{6 6 . 8}$ \\
\hline
\end{tabular}

compared with the original SSD, respectively. The reason why the class 'airplane' obtains 100 percentage in all the four models is that, there is only 1 airplane object in the test dataset of VEDAI
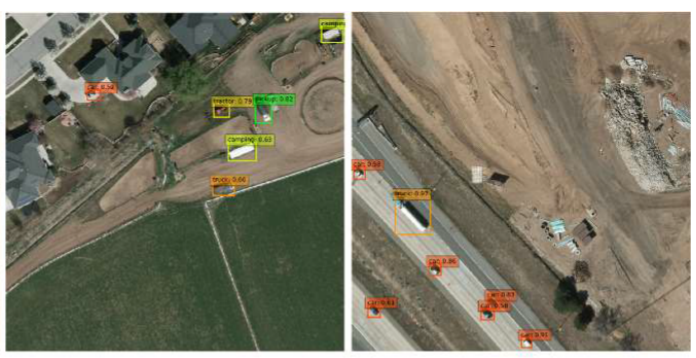

(a)
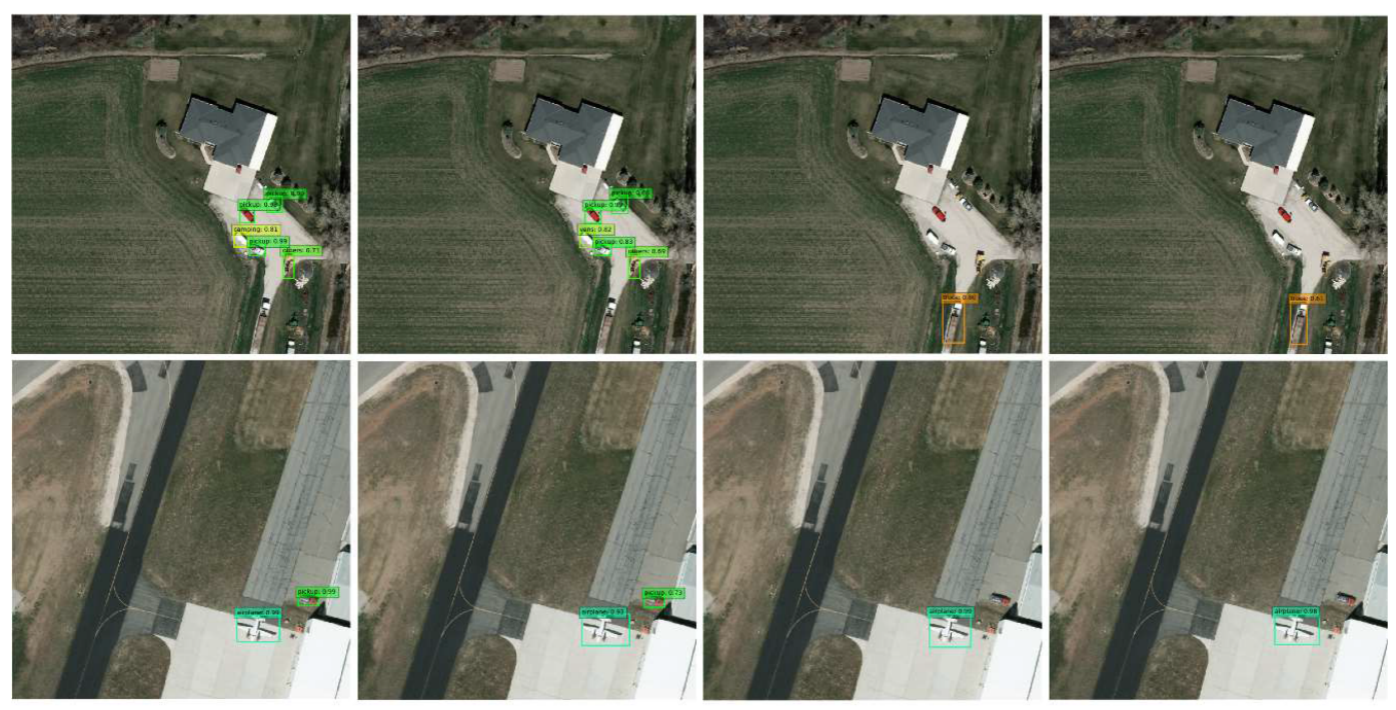

(b)

Figure 3: Visualization of the detection results on VEDAI dataset. (a): The first two image are the results of improved SSD-300, and the last two image are the results of improved SSD-512. (b): First column and second column are the detection results of the improved SSD-512 and the improved SSD-300, respectively. The third column and the forth column are the results of the original SSD-512 and the Original SSD-300, respectively. 
and detecting that airplane object is not different for all the four models.

We also compare the performance of our improved SSD with other three SOTA target detection algorithm, Faster RCNN, YOLO V2, YOLO V3, as shown in Table 2. We use the same input size 512 and our improved SSD achieves $66.8 \% \mathrm{mAP}$, outperforming all these three algorithms. For the two-stage detection algorithm Faster RCNN, it achieves better performance than the two onestage algorithm YOLO V2 and YOLO V3, but the shortcoming of the two-stage method is the speed, which is a very important index to evaluate the practicability. Our improved SSD as a one-stage method not only achieves better mAP than the two-stage method Faster RCNN but also has advantage on the speed. The experiments results demonstrate that our improved SSD has better performance for small targets detection.

\subsection{Qualitative Results}

To make the improvements of our improved SSD be visualized, we make a qualitative comparison between the proposed improved SSD and the original SSD on VEDAI dataset. As shown in Figure 3(a), the first two image are the results of improved SSD-300, and the last two image are the results of improved SSD-512. There are several small targets which are surrounded by complexed background, and our proposed detector is able to detect the various vehicles in the pictures. The result obtained by the model with $512 \times 512$ input size is outperforming than the one with $300 \times 300$ input size since the high resolution input images provide more semantic details which is of great importance for the classification.

Besides, the proposed detector can detect the targets which are missed by the original SSD, as shown in Figure 3(b). The first column and second column are the detection results of the improved SSD512 and the improved SSD-300, respectively. The third column and the forth column are the results of the original SSD-512 and the Original SSD-300, respectively. There are at least seven small targets in the first image and our improved SSD can detect five of them, but the original SSD could detect only one of them. The second image is more obvious that the two original SSD with different input resolution all ignore the red 'pickup' behind the 'airplane' but our two improved SSD detect it successfully.

The quantitative results and the qualitative results indicates that the improved SSD has better performance on small targets detection than the original SSD.

\section{CONCLUSION}

In this paper, we proposed an improved SSD for small target detection. The main contributions of our network are as follows: First, we include the Conv3_3 feature which has higher resolution as one of source features to make the original SSD more suitable for small targets detection. Second, to enrich the information of the shallow source features, a feature pyramid which is fused by the source features is adopted. What is more, to make a features norm balance between the source features and make new detector more easily to converge, we add a L2 normalization at the top of the Conv3_3 prediction branch. Finally, to verify the performance of the proposed model, we do comparative experiments on VEDAI dataset. The quantitative results and the qualitative results show that our method obtained better performance than the original SSD for small targets detection.

\section{REFERENCES}

[1] L. Mou and X. X. Zhu, 2016. Spatiotemporal scene interpretation of space videos via deep neural network and tracklet analysis," in IEEE International Geoscience and Remote Sensing Symposium (IGARSS)

[2] G. Kopsiaftis and K. Karantzalos, 2015. Vehicle detection and traffic density monitoring from very high resolution satellite video data," in IEEE International Geoscience and Remote Sensing Symposium (IGARSS).

[3] R. Girshick, J. Donahue, T. Darrell, and J. Malik, 2014. Rich feature hierarchiesfor accurate object detection and semantic segmentation. in Proc. IEEE Conf. Comput. Vis. Pattern Recognit. (CVPR), Columbus, OH, USA, pp. 580-587.

[4] R. Girshick, 2015. Fast R-CNN. in Proc. IEEE Int. Conf. Comput. Vis. (ICCV), Dec. 2015, pp. 1440-1448.

[5] S. Ren, K. He, R. Girshick, and J. Sun, 2017. Faster R-CNN: Towards real time object detection with region proposal networks. IEEE Trans. Pattern Anal. Mach. Intell., vol. 39, no. 6, pp. 1137-1149.

[6] W. Liu et al. SSD: 2016. Single shot multibox detector. in Computer Vision ECCV. Springer, pp. 21-37.

[7] J. Redmon, S. Divvala, R. Girshick, and A. Farhadi, 2016. You only look once: Unified, real-time object detection, in Proc. IEEE Conf. Comput. Vis. Pattern Recognit. (CVPR), pp. 779-788.

[8] J. Redmon and A. Farhadi, 2017. YOLO9000: Better, faster, stronger. in Proc. IEEE Conf. Comput. Vis. Pattern Recognit. (CVPR), pp. 6517-6525.

[9] P. Sermanet, D. Eigen, X. Zhang, M. Mathieu, R. Fergus, and Y. Lecun. 2013. Overfeat: Integrated recognition, localization and detection using convolutional networks. [Online]. Available: https://arxiv.org/abs/1312.6229.

[10] Lin. T. Y and Dollar. P, 2017. Feature Pyramid Networks for Object Detection, in Proc. IEEE Conf. Comput. Vis. Pattern Recognit. (CVPR), pp. 936-944.

[11] Y. Fu and W. Liu, 2017. DSSD: Deconvolutional Single Shot Detector. arXiv:1701.06659 [cs.CV]. [Online]. Available: https://arxiv.org/abs/1701.06659

[12] Liu. S, Qi. L and H. Qin, 2018. Path Aggregation Network for Instance Segmentation, in Proc. IEEE Conf. Comput. Vis. Pattern Recognit. (CVPR), pp. 8759-8768.

[13] S. Razakarivony and F. Jurie. 2016. Vehicle detection in aerial imagery: A small target detection benchmark. Journal of Visual Communication and Image Representation. Vol.34, pp.187-203.

[14] K. Simonyan and A. Zisserman, 2015. Very Deep Convolutional Networks for Large-Scale Image Recognition. arXiv:1409.1556 [cs.CV]. [Online]. Available: https://arxiv.org/abs/1409.1556v6

[15] W. Liu, A. Rabinovich and A. C. Berg, 2015. ParseNet: Looking Wider to See Better. arXiv:1506.04579 [cs.CV]. [Online]. Available: https://arxiv.org/abs/1506.04579 